\title{
Statistical Restoration Techniques on Melanoma Images
}

\author{
${ }^{1}$ Reshma. M, ${ }^{2}$ Dr. B Priestly Shan \\ ${ }^{1}$ Assistant Professor, Dept of E\&C, UBDT College of Engg, Davangere, Karnataka \& Research Scholar, \\ Sathyabama University, Chennai \\ ${ }^{2}$ Principal, Eranad Knowledge City-Technical Campus, Manjeri, Kerala \\ Email:reshma.m03@gmail.com,priestlyshan@gmail.com
}

Received: 22 ${ }^{\text {nd }}$ October 2018, Accepted: $28^{\text {th }}$ November 2018, Published: $31^{\text {st }}$ December 2018

\begin{abstract}
In this paper, a comparative study of image restoration on color images of skin cancer is presented as it plays an important role in the study of images. Image restoration is the process of convalescing an image from a tarnished version - usually a blurred and noisy image. Image restoration is an elementary problem in image processing, and it also provides a test bed for more universal converse problems. Key issues that must be addressed are the quality of the restored image, the computational efficiency of the algorithm, and the performance parameters such as the Peak signal to noise ratio (PSNR), Mean square error (MSE). Image noise is unwanted signal which comes in image from sensor such as thermal or electrical signal and Environmental condition such as rain, snow etc. Researchers have proposed many methods in this regard and in this paper we will examine and discuss different restoration methods and performance parameters for Melanoma images.
\end{abstract}

\section{Keywords}

Peak Signal to Noise Ratio, Mean Square Error, Median Filter

\section{Introduction}

Skin diseases are the most common diseases in humans and its frequency is ever-increasing noticeably. Therefore early diagnosing the skin cancer is a crucial issue for patient. However, a highly qualified and skilled doctor is able to categorize the skin cancer from other normal skin diseases. Therefore it is very essential to develop the computer based skin cancer detection so that it would become easy for non-specialized doctors to identify the disease. It is wellknown that early detection of skin cancer and its treatment can reduce the mortality and morbidity of patients. Digital Dermoscopy is one of the most extensively used methods to identify and classify skin-cancer. An automatic medical images analysis system has usually three stages: (1) Proper restoration \& Enhancement, (2) feature extraction and selection (3) Classification. The proper restoration is the most important, since it affects the precision of the subsequent steps.

\section{Image Restoration}

Image-processing may be categorized into three functional types: Image Restoration, Image Enhancement and Information Extraction. The description of each of the type is given below[1]:

a. Image Restoration: It helps in reducing data errors, noise and geometric distortions which might have occurred during the scanning, recording, and playback operations. Some of the restoring are as below:

- $\quad$ Restoring periodic line dropouts

- Restoring periodic line striping

- Filtering of random noise

- Correcting for atmospheric scattering

- correcting geometric distortions

b. Image Enhancement: It is an approach of altering the visual impact of an image on the interpreter in a fashion that improves the information content. Some of the most commonly used image enhancement are as listed below:

- Contrast enhancement

- Intensity, hue, and saturation transformations

- Density slicing

- Edge enhancement

- Digital mosaics

- Synthetic stereo images

c. Information Extraction: This helps in decision-making capability of the computer to recognize and classify pixels on the basis of their digital signatures. Some of the most frequently used methods are:

- Principal-component images

- Ratio images 
- Multispectral classification

- Change-detection images

Image restoration is objective whereas the image enhancement is subjective and the latter is designed to put emphasis on features of the image and make the image more pleasing to the observer. Most image enhancement techniques provided by imaging packages use no a priori model of the process that created the image. Image enhancement process effectively removes the noise by sacrificing some resolution which is not acceptable in most of the applications. An image restoration technique reduces noise and recovers resolution loss. Image processing techniques mainly performed either in the spatial domain or the frequency domain. The most basic and a conservative technique for image restoration is deconvolution, whose process is carried out in the frequency domain. In this process, Fourier transform is computed for both the image and the PSF and also it will recover the resolution loss caused by the various blurring factors[10].

The deconvolution technique has a major demerit of poor matrix condition number because of its direct inversion of the PSF which in turn amplifies noise and creates an flawed deblurred image. Typically the blurring process is implicitly considered as shift-invariant and hence more refined techniques, such as regularized de-blurring, have been developed to offer strong recovery under different types of noises and blurring functions. Image restoration is different from Image Enhancement as image restoration is purely depending on mathematical models and Enhancement is based on human perception. Image Enhancement could not exactly represent by mathematical model whereas in Image restoration is related to feature extraction from the imperfect image. Enhancement is manipulated the degraded image, increases the contrast of the image and visual appearance can be improved.

\section{Reasons for Occurrences of Degradation}

There are many reasons available for degradation such as sensor noise, camera-mis-focus, relative object-camera motion, random atmospheric turbulence. Random variation of brightness or color information in the image is called noise as it can be produced by sensor and circuitry of a scanner or digital camera. Also motion blur can be caused while object moves to the camera or vice versa. Another example of image blur is while the object is out of focus of the camera during exposure. This kind of blur is called defocus blur imaging system is affected by atmospheric turbulence by virtue of wave propagation through a medium with non-uniform index of refraction. The situation in which degradation occurs is image acquisition and transmission of the image. Types of distortions[2] are space variant and space invariant where all pixels are suffered from the same distortion problem is space invariant degradation and the distortion suffered by pixels in the image are depending upon their location called space invariant . Space invariant occurred due to camera motion or global lack of focus. Space-variant distortion is complex as it depends on their location with compare to space invariant.

\section{Restoration Techniques}

The various restoration techniques and spatial domain filter are mainly used for noise removal. In spatial domain methods, the image processing techniques operates directly on the picture elements of an image and solely used for the removal of additive noise. Sometimes blur helps to increase photo's articulateness but it reduces the quality of image accidentally[9]. In image restoration, the quality of the restored image is improved over the recorded blurred one and is measured in terms of signal-to-noise ratio. Image restoration techniques are used to make the tarnished image as similar as that of the original image. Some of the statistical image restoration methods are listed as: Median filter, Adaptive filter, Linear filter, IBD (Iterative Blind Deconvolution), NAS-RIF (Nonnegative and Support Constraints Recursive Inverse Filtering), Super-resolution restoration algorithm Based on gradient adaptive interpolation, Deconvolution Using a Sparse Prior, Block-matching, LPA-ICI algorithm, Wiener filter, Deconvolution using Regularized Filter (DRF), Lucy- Richardson Algorithm Techniques, Neural Network Approach[5].

Median Filter: Mean filter is used to remove the salt and pepper noise both simultaneously from the image. We consider a image area of size $\mathrm{m}^{*} \mathrm{n}$ centered at $(\mathrm{X}, \mathrm{Y})$. We find out the mean value of that sub image area and replaces the mean value with the central value. During this process, the image details are not preserved and some details are lost. In this technique to provide simple to use and easy to implement opposite side any unwanted value of the pixel can strongly affected the mean value of all the neighborhood pixels. With the help of the scanning window degraded image pixels are scanned in horizontal and vertical directions. In each pixel scan, the pixel value reappears by the mean value of the scanning window pixels[6]. Median filters can do excellent job of rejecting certain types of noise, in particular, shot or impulse noise in which some of the pixels have intense values. The SUMF is a sliding window spatial filters, but it replaces the centre value in the window with the median of all the pixel values in the window.

Wiener Filter: The backwards separating is a reclamation method for deconvolution, i.e., when the picture is obscured by a known lowpass channel, it is conceivable to recoup the picture by reverse sifting or summed up opposite sifting. Be that as it may, converse sifting is exceptionally touchy to added substance clamor. The methodology of decreasing one corruption at once enables us to build up a rebuilding calculation for each kind of debasement and essentially join them. The Wiener separating executes an ideal tradeoff between reverse sifting and commotion smoothing. It 
evacuates the added substance clamor and rearranges the obscuring all the while. The Wiener filter is a linear estimation of the original image[7].

Gaussian Filter: Gaussian channel is a non-uniform low pass filter. Gaussian separating is utilized to evacuate commotion and detail Gaussian sifting is more powerful at smoothing pictures. It has its premise in the human visual observation framework It has been discovered that in the human visual recognition framework that neurons make a comparative channel when handling visual pictures[3]. This filter considered as the most ground-breaking among the smoothing channels. The Gaussian channel is done by convolving each point in the information cluster with a Gaussian part and after that summing to result in the yield exhibit. The Gaussian channel performs bolster in 8-bit or 32-bit skimming point organizations. For the Gaussian obscure the at first two parameters give the width and stature of the channel window; the (discretionary) third parameter demonstrates the sigma esteem (half width at half max) of the Gaussian piece. On the off chance that the third parameter isn't determined, at that point the Gaussian will most likely be naturally recognized from the window estimate utilizing the following equations.

$\sigma_{\mathrm{x}}=\left(\mathrm{n}_{\mathrm{x}} / 2-1\right) \cdot 30+.80, \mathrm{n}_{\mathrm{x}}=$ param 1

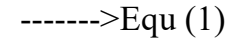

$\sigma_{\mathrm{y}}=\left(\mathrm{n}_{\mathrm{y}} / 2-1\right) \cdot 30+.80, \mathrm{n}_{\mathrm{y}}=$ param 2

Gaussian smoothing develops a weighted mean of each pixel and in addition their neighboring components[8]. The weighting conveys two components, the first in which there is a comparative weighting used by Gaussian smoothing. The second component is and additionally Gaussian weighting anyway found not on the spatial range from the focal pixel. Gaussian clamor has a Gaussian appropriation which has ringer formed likelihood conveyance work given by:

$\mathrm{G}_{\sigma}(\mathrm{x}, \mathrm{y})=\left(1 /\left(\sqrt{ } 2 \prod \sigma^{2}\right) \cdot \mathrm{e}^{-((\mathrm{x} 2+\mathrm{y} 2) / 2 \sigma 2))}=\left(1 /\left(\sqrt{ } 2 \prod \sigma\right) \cdot \mathrm{e}^{-(\mathrm{x} 2) / 2 \sigma 2))} \cdot\left(1 /\left(\sqrt{ } 2 \prod \sigma\right) \cdot \mathrm{e}^{-(\mathrm{y} 2) / 2 \sigma 2)}\right.\right.\right.$

Unsharp Filter: An unsharp covering channel is otherwise called edge upgrade channel. The name was given in light of the fact that the way that the unsharp covering channel enhances subtle elements and other high recurrence segments in edge zone by means of a procedure by subtracting a handled picture from unique picture. Therefore, the unsharp concealing channel is for the most part utilized in the printing ventures or photographic for improving points of interest. The unsharp concealing calculation can be depicted by the condition: $v=y+(x-y)$ where $x$ is the info picture, $y$ is the consequence of a straight low-pass channel, and the gain $\gamma(\gamma>0)$ is a genuine scaling factor. The detail flag $d=(x-y)$ is more often than not opened up to expand the sharpness. Nonetheless, the flag contains 1$)$ points of interest of the picture, 2) clamor, and 3) over-shoots also, under-shoots in zones of sharp edges due to the smoothing of edges.

While the improvement of clamor is plainly bothersome, the improvement of the under-shoot and over-shoot makes the outwardly disagreeable radiance impact. In a perfect world, the calculation should just improve the subtle elements of an picture. Because of this reason we necessitate that the channel is not delicate to clamor and does not smooth sharp edges. The edge protecting channels, for example, nonlinear channels, cubic channel and are utilized to supplant the direct low-pass channel. The previous is less delicate to clamor and the last does not smooth sharp edges. To decrease the corona impact, edge-safeguarding channels such as: versatile Gaussian channel, weighted slightest squares based channels, non-nearby means channel, and two-sided channels are utilized. An essential issue related with the unsharp covering calculation is that the outcome is for the most part out of the range. For precedent, for a 8bit picture, the range is $[0,255]$. A cautious rescaling process is generally required for each picture.

\section{Performance Parameter}

Comparing restoration results requires a measure of image quality. Two commonly used measures are Mean-Squared Error and Peak Signal-to-Noise Ratio.

Peak Signal to Noise Ratio (PSNR) :PSNR is the ratio between maximum possible power of a signal and the power of distorting noise which affects the quality of its representation. It is defined by:

$\mathrm{PSNR}=10 . \log _{10}\left[\mathrm{MAX}^{2} / \mathrm{MSE}\right]$

$\mathrm{PSNR}=20 \cdot \log _{10}[\mathrm{MAX} / \sqrt{\mathrm{MSE}}]$

$\mathrm{PSNR}=20 . \log _{10}(\mathrm{MAX})-10 . \log _{10}[\mathrm{MSE}]$

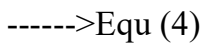

Where MAX maximum signal value that exists is in our original image $M S E$ is root mean square error.

Mean Square Error (MSE) The MSE is the cumulative square error between the encoded and the original image defined by: $\quad M S E=\frac{1}{m n} \sum_{i=0}^{m-1} \sum_{j=0}^{n-1}[I(i, j)-K(i, j)]^{2}$ 
Where, $\mathrm{I}$ is the original image and $\mathrm{K}$ is the uncompressed image. The dimension of the images is $\mathrm{m} \mathrm{x} \mathrm{n}$. Thus MSE should be as low as possible for effective image quality.

\section{Results and Discussion}

Applied a various restoration methods for a dataset of 10 melanoma images as shown in Fig 1. Fig 2, Fig 3, Fig 3, Fig 4, Fig 5 represents a results of restored images on single Melanoma images for Wiener filter, Median filter, Gaussian filter, Unsharp channel individually with introduction of Gaussian noise and salt and pepper noise[4]. The outcomes were checked utilizing PSNR and MSE counts[11]. PSNR is the ratio of maximum power of the signal to the power of the noise which is corrupted, which affects the fidelity of representation[12]. MSE is utilized to find out the event of undesirable clamor in the picture. In Table 1 MSE values are calculated. Lower the MSE values better removal of noise. On comparing these MSE values we came to know that unsharp and wiener filter are having less value. So that is considered to be the better filters for noise removal. From Table 2 we see that median filter is having a higher value where the restored image is having a better image quality refer Fig 3. Fig 6, Fig 7 shows the graphical analysis for different parameter values. Results are displayed for a particular real time image and the estimation of PSNR and MSE is figured for various images utilizing MATLAB.

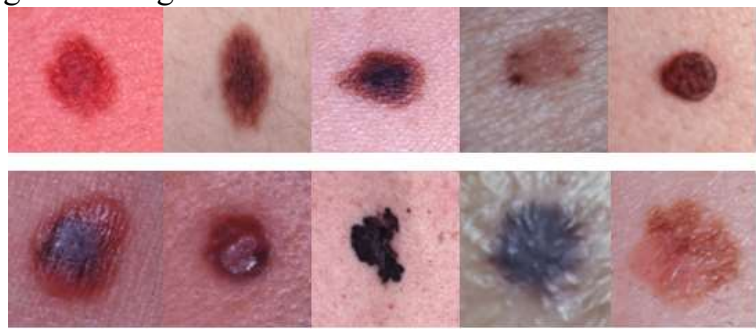

Fig 1: Dataset of 10 Melanoma Images

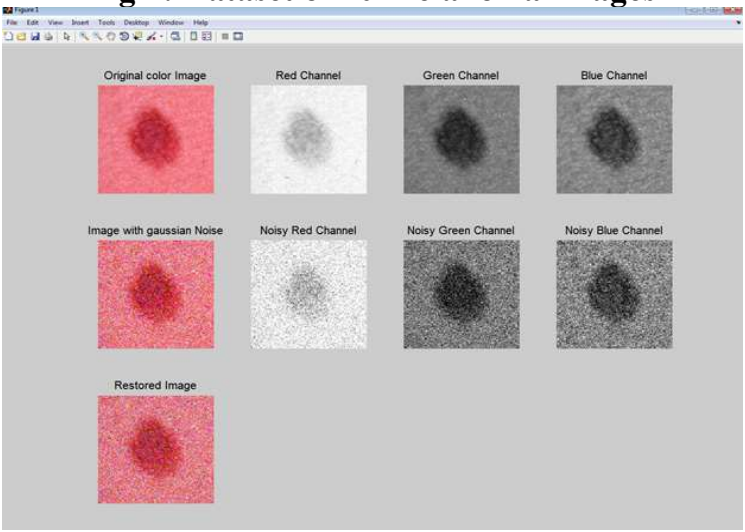

Fig 2: Wiener Filter for Single Image

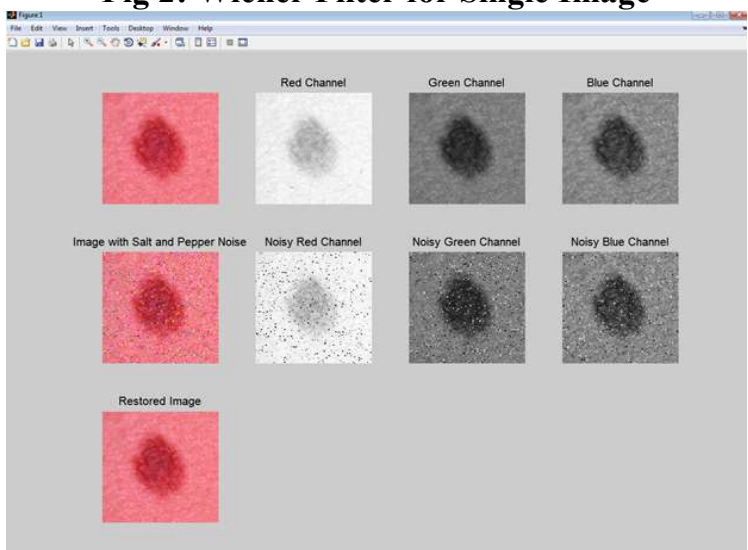

Fig 3: Median Filter for Single Image 


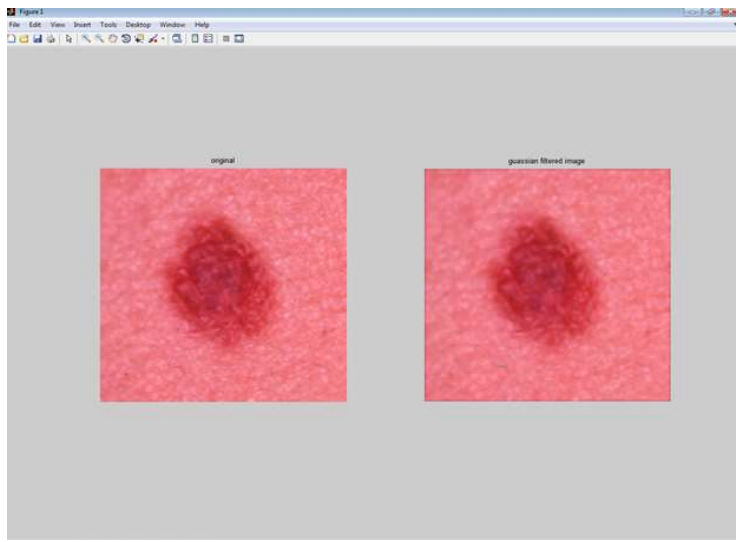

Fig 4: Gaussian Filter for Single Image

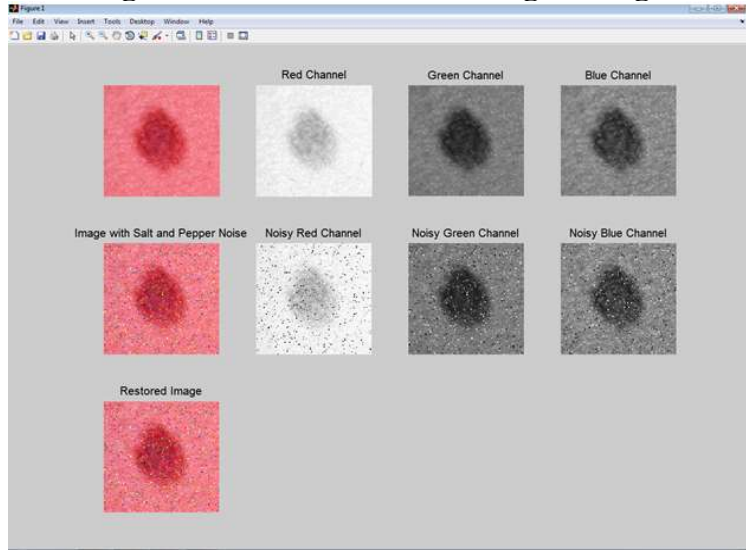

Fig 5: Unsharp Filter for Single Image

\begin{tabular}{|c|c|c|c|c|}
\hline Images & Weiner & Median & Gaussian & unsharp \\
\hline $1 . b m p$ & $1.34 \mathrm{e}+03$ & 3.073 & 6.9 & $1.62 \mathrm{e}+03$ \\
\hline $2 . b m p$ & $1.39 \mathrm{e}+03$ & 2.688 & 6.88 & $2.997 \mathrm{e}+03$ \\
\hline $3 . b m p$ & $1.24 \mathrm{e}+03$ & 6.323 & 9.65 & $3.23 \mathrm{e}+03$ \\
\hline $4 . b m p$ & $1.56 \mathrm{e}+03$ & 4.403 & 6.02 & $3.64 \mathrm{e}+03$ \\
\hline $5 . b m p$ & $1.34 \mathrm{e}+03$ & 3.756 & 7.00 & $2.59 \mathrm{e}+03$ \\
\hline $6 . b m p$ & $1.47 \mathrm{e}+03$ & 2.532 & 6.41 & $4.255 \mathrm{e}+03$ \\
\hline $7 . b m p$ & $1.48 \mathrm{e}+03$ & 3.801 & 6.52 & $3.22 \mathrm{e}+03$ \\
\hline $8 . b m p$ & $1.28 \mathrm{e}+03$ & 7.105 & 7.63 & $3.202 \mathrm{e}+03$ \\
\hline $9 . b m p$ & $1.57 \mathrm{e}+03$ & 1.456 & 6.38 & $4.87 \mathrm{e}+03$ \\
\hline $10 . \mathrm{bmp}$ & $1.45 \mathrm{e}+03$ & 3.467 & 7.42 & $2.37 \mathrm{e}+03$ \\
\hline
\end{tabular}

Table 1. Comparing MSE for Different Filters

\begin{tabular}{|c|c|c|c|c|}
\hline Images & Weiner & Median & Gaussian & unsharp \\
\hline 1.bmp & 16.85 & 43.25 & 39.79 & 18.00 \\
\hline $2 . b m p$ & 16.69 & 43.83 & 39.78 & 13.66 \\
\hline $3 . b m p$ & 17.17 & 40.12 & 38.35 & 13.03 \\
\hline $4 . b m p$ & 16.18 & 41.68 & 40.3 & 12.51 \\
\hline $5 . b m p$ & 16.83 & 42.38 & 39.71 & 13.99 \\
\hline $6 . b m p$ & 16.44 & 44.09 & 40.16 & 11.84 \\
\hline $7 . b m p$ & 16.40 & 42.32 & 40.07 & 13.04 \\
\hline $8 . b m p$ & 17.03 & 39.61 & 39.33 & 13.07 \\
\hline $9 . b m p$ & 16.31 & 46.50 & 40.1 & 11.29 \\
\hline $10 . b m p$ & 16.49 & 42.73 & 39.45 & 14.38 \\
\hline
\end{tabular}

Table 2. Comparing PSNR for Different Filters 


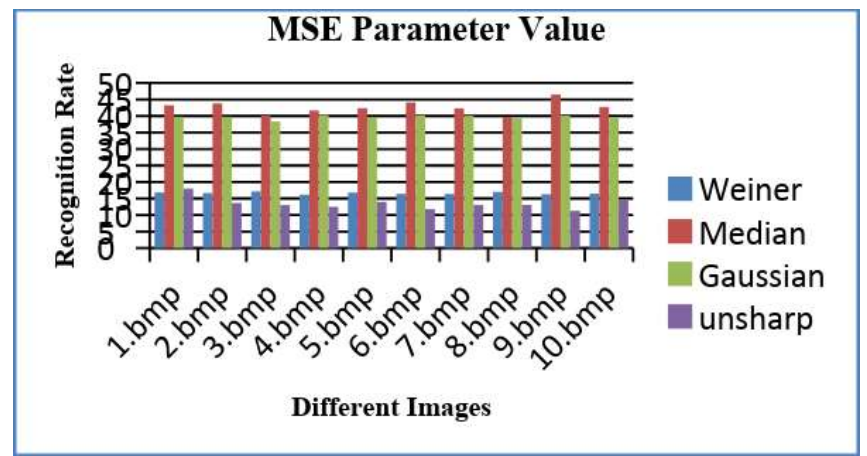

Fig 6. MSE Parameter Values for 10 Different Images

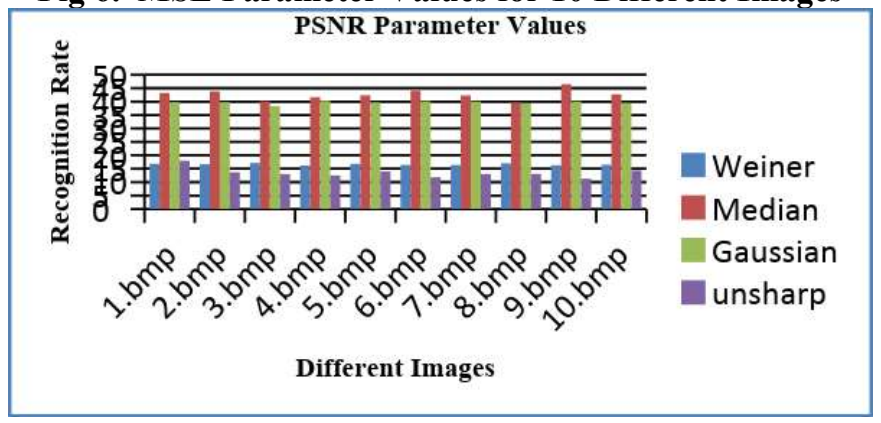

\section{Conclusion}

Fig 7. PSNR Parameter Values for 10 Different Images

In this paper, the observations are made by applying statistical filters on different real time images. Determined different image quality metrics and by estimating these values analyzed the improvement in image quality.

\section{References}

[1].A. Maurya and R. Tiwari, "A Novel Method of Image Restoration by using Different Types of Filtering Techniques,” Int. J. Eng. Sci. Innov. Technol., vol. 3, no. 4, pp. 124-129, 2014.

[2].R. Nagayasu, N. Hosoda, N. Tanabe, H. Matsue, and T. Furukawa, "Restoration method for degraded images using two-dimensional block Kalman filter with colored driving source,” 2011 Digit. Signal Process. Signal Process. Educ. Meet. DSP/SPE 2011 -Proc., pp. 151-156, 2011.

[3].Nachtegeal, Waken. karre, E.E.2005.IEEE Explore Fuzzy filters of noise reduction .The case of Gaussian noise.

[4].Galatsanos, N.P. and R. Chin, "Digital Restoration of Multichannel Images", IEEE Transactions on Signal Processing, vol. 37, pp. 415-421, 1989.

[5].Y. Wang, "Image filtering: noise removal, sharpening, deblurring," http//eeweb.poly.edu/ yao Polytech., p. 41, 2006.

[6].P. Dhole and N. Chopde, "A Comparative Approach for Analysis of Image Restoration using Image Deblurring Techniques,” Int. J. Curr. Eng. Technol., vol. 5, no. 2, pp. 1046-1049, 2015.

[7].Lagendijk, R.L. and J. Biemond, Iterative Identification and Restoration of Images, Kluwer Academic Publishers, Boston, M.A., 1991.

[8].Er. Priya Tiwari, Dr. Naveen Dhillon and Er. Kuldeep Sharma.Vol.3, no. 4,1.July 2013.Analysis of Image Restoration Techniques for Developing Better Restoration Method.

[9].Birwa Raja Mohapatra, Ansuman Mishra, SarahKumarRout.Vol.2,No.3, March 2014 .A Comprehensive Review on Image Restoration Techniques.

[10].R. Kaur and E. N. Singh, "Image Restoration -A Survey," IOSR J. Comput. Eng., vol. 16, no. 4, pp. 107-111, 2014.

[11].Parminder Kaur and Jagroop Singh, "A Study on the Effect of Gaussian Noise on PSNR Value for Digital Images ," International Journal of Computer and Electrical Engineering, Vol. 3, No. 2, April, 2011.

[12]. Pinki and Rajesh Mehra, "Estimation of the Image Quality under Different Distortions," International Journal of Engineering And Computer Science, Volume 5, Issues 7, July 2016. 